\title{
116. The Mathematical Theory of the Electromagnetic Fields in Anisotropic Inhomogeneous Media
}

\author{
By Yoshio HaYashi \\ Department of Mathematics, College of Science \\ and Engineering, Nihon University \\ (Comm. by K. KunUGI, M.J.A., Oct. 12, 1960)
}

Up to this time, theories of electromagnetic field have been studied mainly in isotropic and homogeneous media. Recently the studies of the fields in anisotropic inhomogeneous media have increased their importance in connection with the advances in microwave techniques or in other branches of electric engineerings and physics. But since the rigorous analysis of the fields in such media is so difficult and complicated, it seems to the author that there are few papers on these themes which are rigorous and general enough. In this paper, he will develop the mathematical theory of electromagnetic fields in anisotropic inhomogeneous media in its general form.

1. Any electromagnetic phenomena in a macroscopic scale are represented completely by vector functions $\boldsymbol{E}, \boldsymbol{H}, \boldsymbol{D}, \boldsymbol{B}, \boldsymbol{K}$ and a scalar function $\rho$, which satisfy Maxwell's equation. In addition to the equation, there are three relations between them. In the old theory in isotropic and homogeneous media, they were $\boldsymbol{D}=\varepsilon \boldsymbol{E}, \boldsymbol{B}=\mu \boldsymbol{H}$ and $\boldsymbol{K}=\sigma \boldsymbol{E}$ where $\varepsilon, \mu$ and $\sigma$ are scalar constants characteristic of the medium. In this paper we take them as follows: $\boldsymbol{D}=[\varepsilon] \boldsymbol{E}+[\xi] \boldsymbol{H}$, $\boldsymbol{B}=[\mu] \boldsymbol{H}+[\zeta] \boldsymbol{E}, \boldsymbol{K}=[\sigma] \boldsymbol{E}$, where a roman letter in a bracket, such as $[\varepsilon]$, represents a $3 \times 3$ matrix, the elements $\varepsilon_{i, j}(i, j=1,2,3)$ of which are functions of position. The fact that the characters of the medium are represented by matrices shows that the medium is anisotropic, and that the elements of these matrices are the functions of position shows that the medium is inhomogeneous. Of course these representations include all of the isotropicity and anisotropicity, homogeneity and inhomogeneity of the medium. Because of $[\varepsilon]$ etc., it is not possible to eliminate $\boldsymbol{E}$ or $\boldsymbol{H}$ from Maxwell's equation, hence the old method of analysis is not available. A new method will be investigated in the followings.

Let $\varepsilon, \mu$ and $\sigma$ be arbitrary scalar constants, and put $\varepsilon_{i j}^{\prime}=\varepsilon_{i j}-\varepsilon \delta_{i j}$, $\mu_{i j}^{\prime}=\mu_{i j}-\mu \delta_{i j}, \sigma_{i j}^{\prime}=\sigma_{i j}-\sigma \delta_{i j}$. Suppose [ $\left[\varepsilon^{\prime}\right]$ is a matrix the elements of which are $\varepsilon_{i j}^{\prime}$, then $[\varepsilon]=\varepsilon U+\left[\varepsilon^{\prime}\right]$, where $U$ is the unit matrix. Similar holds for $[\mu]$ and $[\sigma]$. Hence Maxwell's equation will be reduced to

$$
\nabla \times \boldsymbol{E}=-i \omega \mu \boldsymbol{H}-\boldsymbol{K}_{H}, \quad \nabla \times \boldsymbol{H}=(\sigma+i \omega \varepsilon) \boldsymbol{E}+\boldsymbol{K}_{E}
$$

where $\boldsymbol{K}_{H}=i \omega\left(\left[\mu^{\prime}\right] \boldsymbol{H}+[\zeta] \boldsymbol{E}\right)$ and $\boldsymbol{K}_{E}=\left[\sigma^{\prime}+i \omega \varepsilon^{\prime}\right] \boldsymbol{E}+i \omega[\xi] \boldsymbol{H}$, when the 
fields depend on time $t$ through the factor $e^{i \omega t}$ with a complex constant $\omega$. (1) provide the fields in isotropic and homogeneous medium with the medium constants $\varepsilon, \mu$ and $\sigma$, in the presence of the distributions of the densities of electric current $\boldsymbol{K}_{E}$ and magnetic current $\boldsymbol{K}_{H}$. If the time dependence of the fields is more general, the Laplace transformation about $t$ will give $\nabla \times \boldsymbol{E}=-p \mu \boldsymbol{E}-\boldsymbol{K}_{H}, \nabla \times \boldsymbol{H}=(\sigma+p \varepsilon) \boldsymbol{E}+\boldsymbol{K}_{E}$, $\boldsymbol{K}_{H}=p\left(\left[\mu^{\prime}\right] \boldsymbol{H}+[\zeta] \boldsymbol{E}\right)-\boldsymbol{B}_{0}, \boldsymbol{K}_{E}=\left[\sigma^{\prime}+p \varepsilon^{\prime}\right] \boldsymbol{E}+p[\xi] \boldsymbol{H}+\boldsymbol{D}_{0}$, where $p$ is the parameter of the transformation, and $\boldsymbol{D}_{0}, \boldsymbol{B}_{0}$ are the initial values of $\boldsymbol{D}$ and $\boldsymbol{B}$ respectively. These are formally the same as (1), hence we shall study about (1) only in the followings.

If constants $\eta$ and $\kappa$ are defined as follows: $\eta^{2}=-i \omega \mu /(\sigma+i \omega \varepsilon)$, $\kappa=(\sigma+i \omega \varepsilon) \eta$, two equations of (1) will be unified as

$$
\nabla \times \boldsymbol{F}=\kappa \boldsymbol{F}+\kappa \boldsymbol{G}, \quad \nabla \cdot \boldsymbol{F}=-\nabla \cdot \boldsymbol{G}
$$

where $\boldsymbol{F}=\boldsymbol{E}+\eta \boldsymbol{H}$ and $\boldsymbol{G}=\frac{\eta}{\kappa} \boldsymbol{K}_{E}-\frac{\eta}{\eta \kappa} \boldsymbol{K}_{H}$. Separating (2) about $\eta{ }^{*)}$ it reduces to (1), i.e. (1) and (2) are equivalent to each other. Thus we have diminished the number of unknowns.

2. It is desirable to have the integral theorem and the integral representation for the vector $\boldsymbol{F}$ introduced in the preceding section. Let $D$ be a domain, $S$ be its boundary, and $\boldsymbol{n}$ be the outer normal unit vector of $D$ on $S$. Suppose $\psi=-\exp (-i \kappa R) / 4 \pi R$, where $R=\overline{P Q}$ is the distance of two points $P$ and $Q$, and $\stackrel{\circ}{\kappa}$ is one of the roots of $\kappa^{2}$, of which $\operatorname{Im} \AA<0$.

Theorem 1. Assume that i) $S$ is a sum of finite number of regular Jordan closed surfaces. ii) $\boldsymbol{F}$ and $\boldsymbol{G}$ are continuous functions in $D+S$, with the continuous bounded partial derivatives of the first order in $D$ and satisfy (2) there. Then

$$
\begin{aligned}
\boldsymbol{F}(P) & =\int_{S} \boldsymbol{U}(P, Q) d S_{Q}-\int_{D} \boldsymbol{V}(P, Q) d V_{Q}, \quad(P \in D) \\
O & =\int_{S} \boldsymbol{U}(P, Q) d S_{Q}-\int_{D} \boldsymbol{V}(P, Q) d V_{Q}, \quad(P \notin D+S) \\
\Omega / 4 \pi \quad \boldsymbol{F}(P) & =\int_{S}^{*} \boldsymbol{U}(P, Q) d S_{Q}-\int_{D} \boldsymbol{V}(P, Q) d V_{Q}, \quad(P \in S)
\end{aligned}
$$

where $*$ indicates that the integral is taken in the sense of Cauchy's principal value of a singular integral, $\Omega$ is the solid angle at $P$ intercepted by $S$, and

$$
\begin{gathered}
\boldsymbol{U}(P, Q)=-\frac{1}{\kappa} \nabla_{Q} \cdot[\boldsymbol{n} \times \boldsymbol{F}]_{Q} \nabla_{Q} \psi+[\boldsymbol{n} \times \boldsymbol{F}]_{Q} \\
\quad \times \nabla_{Q} \psi+\kappa \psi[\boldsymbol{n} \times \boldsymbol{F}]_{Q}-(\boldsymbol{n} \cdot \boldsymbol{G})_{Q} \nabla_{Q} \psi \\
\boldsymbol{V}(P, Q)=-(\nabla \cdot \boldsymbol{G})_{Q} \nabla_{Q} \psi+\kappa \boldsymbol{G}_{Q} \times \nabla_{Q} \psi+\kappa^{2} \psi \boldsymbol{G}_{Q} .
\end{gathered}
$$

*) M. Itoh: Properties of complex oscillating electromagnetic field and unified equation for electromagnetic oscillation, Trans. I. E. E. Japan, 3, no. 4 (1942). 
Theorem 2. Under the same conditions as in Theorem 1,

$$
\begin{gathered}
\int_{S}[\boldsymbol{n} \times \boldsymbol{F}]_{Q} \cdot \nabla_{Q} \psi d S_{Q}=\int_{S} \kappa \boldsymbol{n} \cdot(\boldsymbol{F}+\boldsymbol{G})_{Q} \psi d S_{Q}=-\int_{S} \psi \nabla_{Q}^{(2)} \cdot[\boldsymbol{n} \times \boldsymbol{F}]_{Q} d S_{Q} \\
\begin{aligned}
\int_{S}\left([\boldsymbol{n} \times \boldsymbol{F}]_{Q} \cdot \nabla_{Q}\right) \nabla_{Q} \psi d S_{Q} & =\int_{S} \kappa \boldsymbol{n} \cdot(\boldsymbol{F}+\boldsymbol{G})_{Q} \nabla_{Q} \psi d S_{Q} \\
& =-\int_{S} \nabla_{Q}^{(2)} \cdot[\boldsymbol{n} \times \boldsymbol{F}]_{Q} \nabla_{Q} \psi d S_{Q}
\end{aligned}
\end{gathered}
$$

where $\nabla^{(2)}=\nabla-\boldsymbol{n} \frac{\partial}{\partial n}$. If $P \in S, *$ must be taken in the both hand sides.

More general theorems about an arbitrary vector function $\boldsymbol{v}$ and a scalar wave function $\varphi$ can be proven, by the help of Gauss' divergence theorem, from which we can deduce Theorems 1 and 2, by replacing $\boldsymbol{v}$ and $\varphi$ with $\boldsymbol{F}$ and $\psi$ respectively. The grad. of (4) will be (5), and conversely the div. of (5) will be (4). Therefore (4) and (5) are equivalent to each other. The importance of (4) and (5) exists in that the singularity of the integrand in the first term of these equations at $P=Q$ is lowered by the second and third terms of them. (2) and (3) are a generalization of Cauchy-Riemann equation and Cauchy's integral representation in the theory of two dimensional complex function respectively. In fact, it will be proven that the former reduces to the latter when we assume that $\boldsymbol{G}=\mathbf{0}$ (this means that the medium is isotropic and homogeneous) $\partial / \partial z=0, F_{z}=0$ (this means that the field is two dimensional) and $\kappa=0$ (this means that $\omega=0$, or that the field is static one). Thus our theory involves the theory of function of two dimensional complex variable as its special case, and makes a function theoretic investigation of the electromagnetic fields.

Theorem 3. (6) $\quad \tau_{P} \equiv \Gamma_{P}^{(2)} \cdot[\boldsymbol{n} \times \boldsymbol{F}]_{P}+\kappa \boldsymbol{n} \cdot(\boldsymbol{F}+\boldsymbol{G})_{P}=0 . \quad(P \in S)$

From (4), $\phi_{P} \equiv \int_{S} \tau_{Q} \psi d S_{Q}=0(P \notin S)$. Being continuous, $\phi_{P}=0$ everywhere, hence $\nabla_{P} \phi_{P}=0$. But $\nabla_{P} \phi_{P}$ is discontinuous on $S$, the amount of which is $\tau_{P}$. Therefore $\tau_{P}=0$. Conversely, we shall have (4) from (6) easily. Thus we have proven not only (6) but the equivalence of (4) and (6).

Theorem 4. Let $\boldsymbol{J}$ be an arbitrary tangential vector defined on $S$, and $-\varpi=V \cdot J$. Suppose $\boldsymbol{G}$ is an arbitrary vector function continuous in $D+S$ with continuous partial derivatives of the first order in $D$. Then the function $\boldsymbol{F}$, defined as

$$
\begin{aligned}
\boldsymbol{F}_{P} & =\int_{S}\left\{\frac{1}{\kappa} \boldsymbol{\varpi}_{Q} \nabla_{Q} \psi+\boldsymbol{J}_{Q} \times \nabla_{Q} \psi+\kappa \psi \cdot \boldsymbol{J}_{Q}-(\boldsymbol{n} \cdot \boldsymbol{G})_{Q} \nabla_{Q} \psi\right\} d S_{Q} \\
& -\int_{D}\left\{-(\nabla \cdot \boldsymbol{G})_{Q} \nabla_{Q} \psi+\kappa \boldsymbol{G}_{Q} \times \nabla_{Q} \psi+\kappa^{2} \psi \boldsymbol{G}_{Q}\right\} d V_{Q}
\end{aligned}
$$


satisfies (2) when $P \in D$, and (8) $\nabla \times \boldsymbol{F}=\kappa \boldsymbol{F}^{\prime}$ when $P \notin D+S$.

Calculation of the limiting values of the function $\boldsymbol{F}$ defined by (7) as $P \notin S$ tends to $P_{0} \in S$, leads to

$$
\begin{aligned}
\boldsymbol{n}_{P_{0}} \times \boldsymbol{F}_{P_{0}}=\lim _{\boldsymbol{P} \rightarrow P_{0}} \boldsymbol{n}_{P_{0}} \times \boldsymbol{F}_{P}=\mp \frac{1}{2} \boldsymbol{J}_{P_{0}} \\
\quad+\int_{S}^{*} \boldsymbol{n}_{P_{0}} \times\left\{\frac{-1}{\kappa} \varpi_{Q} \nabla_{Q} \psi+\boldsymbol{J}_{Q} \times \nabla_{Q} \psi+\kappa \psi \boldsymbol{J}_{Q}-(\boldsymbol{n} \cdot \boldsymbol{G})_{Q} \nabla_{Q} \psi\right\}_{P_{0}} d S_{Q} \\
\quad-\int_{D} \boldsymbol{n}_{P_{0}} \times\left\{-(\nabla \cdot \boldsymbol{G})_{Q} \nabla_{Q} \psi+\kappa \boldsymbol{G}_{Q} \times \nabla_{Q} \psi+\kappa^{2} \psi \boldsymbol{G}_{Q}\right\}_{P_{0}} d V_{Q} .
\end{aligned}
$$

This is a generalization of Plemelj's theorem, from which we shall have

Theorem 5. A) $\frac{1}{2} \boldsymbol{J}_{P_{0}}=\int_{S}^{*} \boldsymbol{n}_{P_{0}} \times\left\{\frac{-1}{\kappa} \varpi_{Q} \nabla_{Q} \psi\right.$

$$
\begin{array}{r}
\left.+\boldsymbol{J}_{Q} \times \nabla_{Q} \psi+\kappa \psi \boldsymbol{J}_{Q}-(\boldsymbol{n} \cdot \boldsymbol{G})_{Q} \nabla_{Q} \psi\right\}_{P_{0}} d S_{Q} \\
-\int_{D} \boldsymbol{n}_{P_{0}} \times\left\{-(\nabla \cdot \boldsymbol{G})_{Q} \nabla_{Q} \psi+\kappa \boldsymbol{G}_{Q} \times \nabla_{Q} \psi+\kappa^{2} \psi \boldsymbol{G}_{Q}\right\}_{P_{0}} d V_{Q}
\end{array}
$$

is the necessary and sufficient condition which $\boldsymbol{J}$ must satisfy, in order that the tangential component $\boldsymbol{n} \times \boldsymbol{F}$ of the function defined in (7) has the limiting value $J$ when $P \in D$ and $P \rightarrow P_{0} \in S$.

B) (8) is the necessary and sufficient condition which $\boldsymbol{J}$ must satisfy in order that the function $\boldsymbol{F}$ defined by (7) vanishes identically when $P \notin D+S$.

3. In this section, simultaneous integral equations will be given, which will determine the components of the field. For the sake of brevity, we shall assume that there are two media separated by a closed surface $S$, the inner medium is anisotropic and inhomogeneous, while the outer one is isotropic and homogeneous, and that the fields satisfy the radiation condition at infinity. Let the domains in the interior and exterior of $S$ be denoted as $D^{-}$and $D^{+}$respectively, and suppose $\boldsymbol{n}$ be the unit normal of $D^{-}$on $S$. Quantities in $D^{ \pm}$, such as the fields and medium constants etc., will be distinguished by suffix \pm , for instance, $\boldsymbol{E}^{ \pm}, \boldsymbol{H}^{ \pm}, \eta^{ \pm}$and $\boldsymbol{\kappa}^{ \pm}$. According to the boundary conditions on $S$, we shall have $\boldsymbol{n} \times \boldsymbol{E}^{-}=\boldsymbol{n} \times \boldsymbol{E}^{+}$(put as $\left.=\stackrel{m}{\boldsymbol{J}}\right), \boldsymbol{n} \times \boldsymbol{H}^{-}=\boldsymbol{n}$ $\times \boldsymbol{H}^{+}(=\stackrel{e}{\boldsymbol{J}}), \varpi_{E}^{+}=-\varpi_{E}^{-}\left(=\varpi_{E}\right), \varpi_{H}^{+}=-\varpi_{H}^{-}\left(=\varpi_{H}\right)$ where $\varpi_{E}^{ \pm}=\mp \nabla \cdot\left[\boldsymbol{n} \times \boldsymbol{E}^{ \pm}\right]$ and $\varpi_{H}^{ \pm}=\mp \nabla \cdot\left[\boldsymbol{n} \times \boldsymbol{H}^{ \pm}\right]$. Moreover, suppose that $\nabla \cdot \boldsymbol{G}_{E}=\frac{\eta^{-}}{\kappa^{-}} \nabla \cdot\left[\sigma^{\prime}+i \omega \varepsilon^{\prime}\right]$ $\boldsymbol{E}^{-}\left(=-\rho_{E}\right)$, and $\nabla \cdot \boldsymbol{G}_{H}=\frac{-i \omega}{\eta^{-} \kappa^{-}} \nabla \cdot\left[\mu^{\prime}\right] \boldsymbol{H}^{-}\left(=-\rho_{H}\right)$. (Assumptions that $[\xi]=[\zeta]=[0]$ and $\boldsymbol{n} \cdot \boldsymbol{G}=0$ on $S$ have been made for the sake of brevity, which do not lose the essentials of analysis.) Then according to Theorem 1, the fields in $D^{ \pm}$will be expressed as follows: 


$$
\begin{array}{lll}
\boldsymbol{E}_{P^{-}}=\int_{S} \boldsymbol{U}_{\bar{E}}^{-}\left(P^{-}, Q\right) d S_{Q}-\int_{D^{-}} \boldsymbol{V}_{\bar{E}}^{-}\left(P^{-}, Q\right) d V_{Q}+\boldsymbol{E}^{-(i)}\left(P^{-}\right) & \\
\boldsymbol{H}_{P^{-}}=\int_{S} \boldsymbol{U}_{\bar{H}}^{-}\left(P^{-}, Q\right) d S_{Q}-\int_{D^{-}} \boldsymbol{V}_{\bar{H}}\left(P^{-}, Q\right) d V_{Q}+\boldsymbol{H}^{-(i)}\left(P^{-}\right), & \left(P^{-} \in D^{-}\right) \\
\boldsymbol{E}_{P^{+}}=\int_{S} \boldsymbol{U}_{E}^{+}\left(P^{+}, Q\right) d S_{Q} & +\boldsymbol{E}^{+(i)}\left(P^{+}\right) \\
\boldsymbol{H}_{P^{+}}=\int_{S} \boldsymbol{U}_{H}^{+}\left(P^{+}, Q\right) d S_{Q} & +\boldsymbol{H}^{+(i)}\left(P^{+}\right), & \left(P^{+} \in D^{+}\right)
\end{array}
$$

where $\boldsymbol{U}_{E}^{ \pm}\left(P^{ \pm}, Q\right)=\mp\left(\frac{\eta^{ \pm}}{\kappa^{ \pm}}-\varpi_{H}(Q) \nabla_{Q} \boldsymbol{\psi}^{ \pm}+\stackrel{m}{\boldsymbol{J}_{Q}} \times \nabla_{Q} \boldsymbol{\psi}^{ \pm}+\eta^{ \pm} \kappa^{ \pm} \boldsymbol{\psi}^{ \pm} \stackrel{e}{\boldsymbol{J}}_{Q}\right)$

$$
\begin{aligned}
& \boldsymbol{U}_{\bar{H}}^{ \pm}\left(P^{ \pm}, Q\right)=\mp\left(\frac{1}{\eta^{ \pm} \kappa^{ \pm}} \varpi_{E}(Q) \nabla_{Q} \boldsymbol{\psi}^{ \pm}+\stackrel{e}{\boldsymbol{J}_{Q}} \times \nabla_{Q} \boldsymbol{\psi}^{ \pm}+\frac{\kappa^{ \pm}}{\eta^{ \pm}} \boldsymbol{\psi}^{ \pm} \boldsymbol{J}_{Q}\right) \\
& \boldsymbol{V}_{\bar{E}}^{-}\left(P^{-}, Q\right)=\left\{\rho_{E}(Q) \nabla_{Q} \boldsymbol{\psi}^{-}+\eta^{-} \kappa^{-} \boldsymbol{G}_{H}(Q) \times \nabla_{Q} \boldsymbol{\psi}^{-}+\left(\kappa^{-}\right)^{2} \boldsymbol{\psi}^{-} \boldsymbol{G}_{E}(Q)\right\} \\
& \boldsymbol{V}_{\bar{H}}^{-}\left(P^{-}, Q\right)=\left\{\rho_{H}(Q) \nabla_{Q} \boldsymbol{\psi}^{-}+\frac{\kappa^{-}}{\eta^{-}} \boldsymbol{G}_{E}(Q) \times \nabla_{Q} \boldsymbol{\psi}^{-}+\left(\kappa^{-}\right)^{2} \boldsymbol{\psi}^{-} \boldsymbol{G}_{H}(Q)\right\}
\end{aligned}
$$

and also $\psi^{ \pm}=-\exp \left(-i{ }^{ \pm} R\right) / 4 \pi R . \quad \boldsymbol{E}^{ \pm(i)}$ and $\boldsymbol{H}^{ \pm(i)}$ are the surface integrals over $S^{ \pm(i)}$ which may exist in $D^{ \pm}$and enclose the sources there. They are assumed to be known functions, which represent the primary incident fields. If $\stackrel{e}{\boldsymbol{J}}, \stackrel{m}{\boldsymbol{J}}, \varpi_{E}$ and $\varpi_{H}$ on $S$, and $\rho_{E}, \rho_{H}, \boldsymbol{G}_{E}$ and $\boldsymbol{G}_{H}$ in $D^{-}$are obtained, (9) will determine the fields in $D^{ \pm}$completely. From these, the simultaneous integral equations about these unknowns can be obtained as follows:

$$
\begin{aligned}
& \boldsymbol{G}_{E}\left(P^{-}\right)=\int_{S} \frac{\eta^{-}}{\kappa^{-}}\left[\sigma^{\prime}+i \omega \varepsilon^{\prime}\right]_{P^{-}} \boldsymbol{U}_{\bar{E}}^{-}\left(P^{-}, Q\right) d S_{Q} \\
& \quad-\int_{D^{-}} \frac{\eta^{-}}{\kappa^{-}}\left[\sigma^{\prime}+i \omega \varepsilon^{\prime}\right]_{P^{-}} \boldsymbol{V}_{\bar{E}}^{-}\left(P^{-}, Q\right) d V_{Q}+\frac{\eta^{-}}{\kappa^{-}}\left[\sigma^{\prime}+i \omega \varepsilon^{\prime}\right]_{P^{-}} \boldsymbol{E}^{-(i)}\left(P^{-}\right) \\
& \boldsymbol{G}_{H}\left(P^{-}\right)=\int_{S} \frac{-i \omega}{\eta^{-} \kappa^{-}}\left[\mu^{\prime}\right]_{P^{-}} \boldsymbol{U}_{H}^{-}\left(P^{-}, Q\right) d S_{Q} \\
& \quad+\int_{D^{-}} \frac{i \omega}{\eta^{-} \kappa^{-}}\left[\mu^{\prime}\right]_{P^{-}} \boldsymbol{V}_{\bar{H}}^{-}\left(P^{-}, Q\right) d V_{Q}-\frac{i \omega}{\eta^{-} \kappa^{-}}\left[\mu^{\prime}\right]_{P^{-}} \boldsymbol{H}^{-(i)}\left(P^{-}\right)
\end{aligned}
$$

$\frac{1}{2}\left(\frac{\kappa^{-}}{\eta^{-}}+\frac{\kappa^{+}}{\eta^{+}}\right) \stackrel{m}{m}_{P}=\int_{S} n_{P} \times \boldsymbol{U}_{E}(P, Q) d S_{Q}-\int_{D^{-}} \frac{\kappa^{-}}{\eta^{-}} n_{P} \times V_{\bar{E}}^{-}(P, Q) d V_{Q}+\boldsymbol{J}_{P}^{(i)}$ $\frac{1}{2}\left(\eta^{-} \kappa^{-}+\eta^{+} \kappa^{+}\right) \stackrel{J}{P}_{P}=\int_{S} \boldsymbol{n}_{P} \times \boldsymbol{U}_{H}(P, Q) d S_{Q}-\int_{D^{-}} \eta^{-} \kappa^{-} \boldsymbol{n}_{P} \times V_{H}^{-}(P, Q) d V_{Q}+\stackrel{\boldsymbol{J}}{P}_{P}^{(i)}$ $\frac{1}{2}\left(\frac{\eta^{-}}{\kappa^{-}}+\frac{\eta^{+}}{\kappa^{+}}\right) \varpi_{H}(P)=\int_{S} \boldsymbol{n}_{P} \cdot \boldsymbol{U}_{E}^{\prime}(P, Q) d S_{Q}-\int_{D^{-}} \boldsymbol{n}_{P} \cdot V_{\bar{E}}^{-}(P, Q) d V_{Q}+\varpi_{H}^{(i)}(P)$ $\frac{1}{2}\left(\frac{1}{\eta^{-} \kappa^{-}}+\frac{1}{\eta^{+} \kappa^{+}}\right) \varpi_{E}(P)=\int_{S} \boldsymbol{n}_{P} \cdot \boldsymbol{U}_{H}^{\prime}(P, Q) d S_{Q}-\int_{D^{-}} \boldsymbol{n}_{Q} \cdot \boldsymbol{V}_{H}^{-}(P, Q) d V_{Q}+\varpi_{E}^{(i)}(P)$ $\frac{1}{3} \sum_{\nu}\left(\sigma_{\nu \nu}+i \omega \varepsilon_{\nu \nu}\right)_{P^{-}} \rho_{E}\left(P^{-}\right)+\frac{1}{3} \eta^{-} \kappa^{-} \sum_{\nu} \boldsymbol{i}_{\nu} \cdot \boldsymbol{G}_{E}\left(P^{-}\right) \epsilon^{\nu p q}\left(\sigma_{p q}^{\prime}+i \omega \varepsilon_{p q}^{\prime}\right)_{P^{-}}$ 


$$
\begin{aligned}
= & -\int_{S} \nabla_{P} \cdot\left[\sigma^{\prime}+i \omega \varepsilon^{\prime}\right]_{P} \boldsymbol{U}_{\bar{E}}^{-}\left(P^{-}, Q\right) d S_{Q}+\int_{D^{-}}\left(\kappa^{-}\right)^{2} V_{P} \cdot\left[\sigma^{\prime}+i \omega \varepsilon^{\prime}\right]_{P} \psi^{-} \boldsymbol{G}_{E}(Q) d V_{Q} \\
& +\int_{D^{-}}\left\{\rho_{E}(Q) \nabla_{Q} \cdot \boldsymbol{X}\left(P^{-}, Q\right)+\eta^{-} \kappa^{-} \boldsymbol{G}_{H}(Q) \cdot \nabla_{Q} \times \boldsymbol{X}\left(P^{-}, Q\right)\right\} d V_{Q} \\
\frac{1}{3} \sum_{\nu}\left(i \omega \mu_{\nu \nu}\right)_{P^{-}} \rho_{H}\left(P^{-}\right)+\frac{1}{3} \frac{\kappa^{-}}{\eta^{-}} \sum_{\nu} \boldsymbol{i}_{\nu} \cdot \boldsymbol{G}_{H}\left(P^{-}\right) \epsilon^{\nu p q}\left(i \omega \mu_{p q}^{\prime}\right)_{P^{-}} & -\int_{\left.\sigma^{\prime}+i \omega \varepsilon^{\prime}\right]_{P} \boldsymbol{E}^{-(i)}\left(P^{-}\right)} \nabla_{P} \cdot\left[i \omega \mu^{\prime}\right]_{P} \boldsymbol{U}_{H}^{-}\left(P^{-}, Q\right) d S_{Q}+\int_{D^{-}}\left(\kappa^{-}\right)^{2} \nabla_{P} \cdot\left[i \omega \mu^{\prime}\right]_{P} \psi^{-} \boldsymbol{G}_{H}(Q) d V_{Q} \\
= & +\int_{D^{-}}\left\{\rho_{H}(Q) \nabla_{Q} \cdot \boldsymbol{X}\left(P^{-}, Q\right)+\frac{\kappa^{-}}{\eta^{-}} \boldsymbol{G}_{E}(Q) \cdot \nabla_{Q} \times \boldsymbol{X}\left(P^{-}, Q\right)\right\} d V_{Q} \\
& -\nabla_{P} \cdot\left[i \omega \mu^{\prime}\right]_{P}-\boldsymbol{H}^{-(i)}\left(P^{-}\right),
\end{aligned}
$$

where $\boldsymbol{U}_{E}=\kappa^{-} / \eta^{-} \boldsymbol{U}_{\bar{E}}^{-}+\kappa^{+} / \eta^{+} \boldsymbol{U}_{E}^{+}, \boldsymbol{U}_{H}=\eta^{-} \kappa^{-} \boldsymbol{U}_{H}^{-}+\eta^{+} \kappa^{+} \boldsymbol{U}_{H}^{+}, \boldsymbol{U}_{E}^{\prime}=\boldsymbol{U}_{E}^{-}+\boldsymbol{U}_{E}^{+}, \boldsymbol{U}_{H}^{\prime}$ $=\boldsymbol{U}_{\boldsymbol{H}}^{-}+\boldsymbol{U}_{\boldsymbol{H}}^{+}$and $\sum \boldsymbol{i}_{\nu} \cdot \boldsymbol{G} \in^{\nu p q} a_{p q} \equiv \boldsymbol{i}_{1} \cdot \boldsymbol{G}\left\{a_{23}-a_{32}\right\}+\boldsymbol{i}_{2} \cdot \boldsymbol{G}\left\{a_{31}-a_{13}\right\}+\boldsymbol{i}_{3} \cdot \boldsymbol{G}\left\{a_{12}\right.$ $\left.-a_{21}\right\} . \quad \boldsymbol{X}$ is a vector, the elements of which are $\chi_{\mu}=\sum_{\nu} \frac{\partial}{\partial x_{\nu}}\left(a_{\nu \mu} \psi^{-}\right)$, where $a_{\nu \mu}$ represent the elements of the matrices $[\sigma],[\varepsilon]$ and $[\mu]$. These quantities, the suffix of which are $(i)$ or $-(i)$, are known functions constructed with $\boldsymbol{E}^{ \pm(i)}$ and $\boldsymbol{H}^{ \pm(i)}$.

It is worth noting that the solutions of these equations determine the fields exactly, because (9) satisfies Maxwell's equations by virtue of Theorem 4, and satisfies the boundary conditions on $S$ by virtue of Theorem 5 and (10). With these simultaneous integral equations of Fredholm type, we shall be able to study about the properties of the fields, for instance the existence or the uniqueness of the solutions. (There is only one term which is yet singular in (10), which will be removed by the use of the Bertrand-Poincare theorem, the result of which is omitted to describe here.) 